\title{
An elliptical-pore model for late-stage planar viscous sintering
}

\author{
By DARREN G. CROWDY \\ Department of Mathematics, Imperial College of Science, Technology and Medicine, \\ 180 Queen's Gate, London SW7 2AZ, UK
}

(Received 12 December 2002 and in revised form 15 August 2003)

A simple 'elliptical-pore model' of the shrinkage of compressible pores in late-stage planar viscous sintering is proposed. The model is in the spirit of matched asymptotics and relies on splitting the flow into an 'inner' and 'outer' problem. The inner problem in the vicinity of any given pore involves solving for its free-surface evolution exactly using complex-variable methods. The outer flow due to all other pores is assumed to be given by an assembly of point sinks/sources. As a test of the model, the evolution of a singly infinite periodic row of compressible pores is considered in detail. The effectiveness of the simple model is tested by comparison with a full numerical simulation. A novel boundary integral method based on Cauchy potentials and conformal mapping is used. In the case of pores with constant pressure, it is found that pores shrink faster than if in isolation. Compressible pores obeying the ideal gas law are also studied and are found to tend to a quasi-steady non-circular state. A higher-order model is also presented and compared with numerical simulations of the viscous sintering of a doubly periodic array of pores in Stokes flow.

\section{Introduction}

Viscous sintering is a process in which a granular compact of particles (e.g. metal or glass) is raised to a sufficiently high temperature that the individual particles become mobile and release surface energy in such a way as to produce inter-particulate bonds (Brinker \& Scherer 1990). At the start of a sinter process, any two particles which are initially touching develop a thin neck which, as time evolves, grows in size to form a more developed bond. As the necks grow in size, the sinter body densifies and any enclosed pores between particles eventually close up. The macroscopic material properties of the compact at the end of the sinter process depend heavily on the degree of densification. In industrial application, it is important to be able to obtain accurate estimates of the time taken for pores to close (or reduce to a sufficiently small size) within any given initial sinter body in order that industrial sinter times are optimized without compromising the macroscopic properties of the final densified material.

While there is an extensive engineering literature on sintering, comparatively few theoretical studies exist and those that do are either phenomenological in nature (e.g. Mackenzie \& Shuttleworth 1952) or are approximate, being based on energy balance arguments with no detailed resolution of the flow fields (e.g. Frenkel 1945). In an attempt to develop a more detailed theory, a sensible first step is to consider the case of planar viscous sintering. A first model of the sintering process is afforded by a 
planar viscous flow in the Stokes regime driven by surface tension effects on the free boundaries between the fluid and the constant-pressure pores.

Numerical methods to compute the evolution of a bounded region of fluid in twodimensional Stokes flow have been devised by Kuiken (1990), Van de Vorst (1993), Pozrikidis (1998) and Primo, Wrobel \& Power (2000). A more realistic model of an extended compact is a doubly periodic array of pores covering the plane. Van de Vorst (1995) and Pozrikidis (2000) have separately devised boundary integral formulations to compute the evolution of such configurations. Formulating the doubly periodic problem presents a number of difficulties. For example, it is natural to restrict computations to a single period cell of the repeated lattice but then the question that immediately arises is how to numerically incorporate the effect of an imposed far-field ambient pressure. Some technical mathematical issues also arise because it is necessary to write the relevant integral representations in a computationally convenient form using Ewald summation techniques (Pozrikidis 2003; Van de Vorst 1993).

There has also been recent interest in the more general problem of computing the pressure in constant-pressure bubbles evolving quasi-steadily in a region of slow, viscous fluid in the Stokes regime (Pozrikidis 2000, 2003). The question of how a constant-pressure enclosure (i.e. a bubble or 'pore') is affected by an ambient viscositydominated flow arises finds natural application in viscous sintering.

At the same time, an increasing number of exact solutions are known for timeevolving surface-tension-driven Stokes flows. Hopper (1990) found an exact solution for the time-dependent coalescence of two equal near-circular cylinders. Richardson (1992) later generalized this to two unequal coalescing cylinders. Extension of Hopper's solutions (Hopper 1990) to multiply connected configurations involving more complicated cylindrical packings has been performed by (Crowdy 2002, 2003a) based on a theory originally presented in Crowdy \& Tanveer $(1998,2001)$ (see also Richardson 2000 for an alternative approach to this problem). In the case of unbounded flows, Antanovskii (1994) and Tanveer \& Vasconcelos (1995) have studied the evolution of an isolated constant-pressure bubble in Stokes flow. These authors find broad classes of exact solutions, including a solution for a constant-pressure, constant-area elliptical bubble evolving in an ambient straining flow with constant strain rate.

Recently, Crowdy (2003b) has extended the exact solutions of Tanveer \& Vasconcelos (1995) to the case of an isolated compressible bubble in an ambient Stokes flow. These results reveal that the exact elliptical solutions of Tanveer \& Vasconcelos (1995) and Antanovskii (1994) can be extended to a compressible elliptical bubble, with non-constant area and pressure, evolving in a straining flow with time-dependent strain rate. These extended solutions form the basis of the model presented in this paper.

The known exact solutions to free-surface Stokes flow problems driven by surface tension usually involve relatively simple flow geometries and their usefulness in studying flows in more complicated geometries has not yet been established. At the same time, full numerical simulations of more complicated flow situations are computationally expensive and frequently hard to formulate. It would therefore seem important to establish whether, and to what extent, simple exact solutions can be harnessed to derive useful and workable models for sintering in complicated flow geometries thereby obviating the need for full numerical investigation. It is the purpose of this paper to propose and test such a model.

The ideas of this paper have been motivated by the elliptical vortex approximation for studying the interaction of vortex patches (Melander, Zabusky \& Styczek 1986; 
Dritschel \& Legras 1991; Saffman 1992; Newton 2001). In two-dimensional vortex dynamics, the elliptical pore approximation assumes that all interacting vortices are ellipses evolving in a time-dependent strain field induced by the other ellipses. This model is predicated on the convenient fact that, according to the dynamics of the Euler equations, an elliptical vortex patch remains elliptical when placed in an ambient, time-dependent straining flow. Such an exact solution was found by Kida (1981) based on extensions of the steady exact solutions of Moore \& Saffman (1971). The present 'elliptical-pore approximation' is predicated on the fact that exactly the same mathematical structure exists in the case of compressible elliptical pores in Stokes flow: a compressible elliptical pore remains elliptical when placed in an ambient time-dependent straining flow, a fact established by Crowdy (2003a).

An elliptical pore approximation is proposed and tested herein. The test-bed problem considered is the shrinkage of an infinite singly periodic row of identical compressible pores equispaced along the $x$-axis. Owing to the nature of the anisotropy of this configuration in the $x$ - and $y$-directions, it is to be expected that initially circular pores will become 'ellipse-like' in shape under evolution. This makes it an ideal case study with which to compare the model. To the best of our knowledge, this configuration has not been considered before by other workers. Consequently, no exact solution to this problem is currently known, nor has it been previously studied numerically.

A second contribution of this paper is to present a novel numerical approach to this class of problems based on ideas frequently employed in plane elasticity but seldom used in the mathematically related problem of Stokes flow. The method relies on a complex-variable reformulation of the problem. The streamfunction is written in terms of two analytic Goursat functions. Integral representations for these functions in terms of Cauchy integrals are then found using techniques from conformal mapping theory to take account of the periodic nature of the flow. The method possesses a number of conceptual advantages over previously employed methods; in particular, it clearly demonstrates the effect on the free-surface dynamics of an imposed (possibly time-dependent) ambient pressure $p_{\infty}(t)$ as well as the effect of making different assumptions for the equation of state in the compressible pore.

While the elliptical pore model is the most generic case, if the pore arrangement has a high degree of spatial symmetry then a 'higher-order model' may be more appropriate. This is the case of a doubly periodic array of pores, for example. In $\S 7$, some higher-order models are also presented and compared with numerical simulations of square and hexagonal arrays performed recently by Pozrikidis (2003).

\section{The elliptical-pore model}

In 'early-stage sintering' the sinter compact consists of a packing of near-circular particles joined by thin interparticle necks. These early-stage processes are modelled well by the various exact solutions of Hopper (1990), Richardson (1992, 2000), and Crowdy $(2002,2003 a)$. Here we are interested in 'late-stage sintering' where the compact has developed into a contiguous region of fluid containing a number of enclosures or 'pores'. A well-known model of late-stage sintering is that of Mackenzie \& Shuttleworth (1952) who propose a phenomenological model where all pores are assumed spherical. Hill (2001) has also considered the problem of the sinter kinetics of a collection of interacting pores during the late stages of viscous sintering. In the latter model, the pores are again assumed to remain spherical under evolution. 

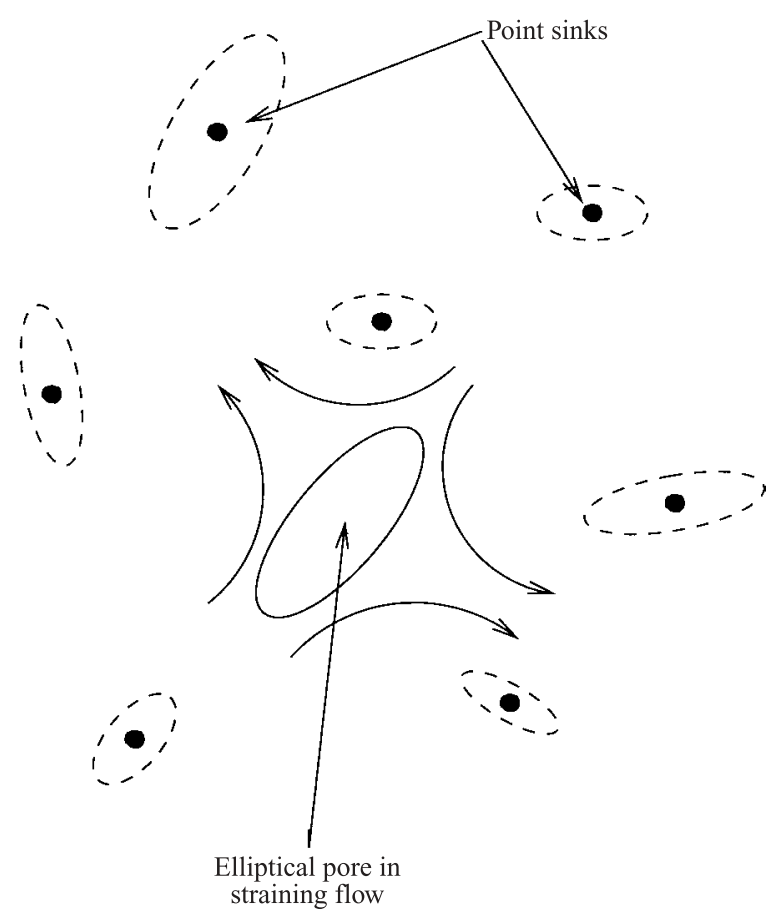

FIGURE 1. Schematic illustrating the 'elliptical pore model' of late-stage viscous sintering. Each pore is modelled as an elliptical bubble in an ambient Stokes flow. For any given pore, the leading-order effect of the presence of all other pores is a local straining flow produced by a distribution of point sinks.

Here, a different model for late-stage pore shrinkage in two-dimensional sintering is presented based on ideas from matched asymptotics. The idea is that close to any given pore, an 'inner problem' is solved using as a far-field boundary condition the inner limit of an appropriate 'outer problem'. At the same time, microstructural information on pore shrinkage rates obtained from the solution to each inner problem gives the required macroscopic parameters for the outer flow approximation. Antanovskii (1996) adopted a similar approach based on matched asymptotic expansions in considering the free-surface evolution of a single constant-pressure bubble in the flow field of Taylor's four-roller mill.

Consider a collection of $N$ pores as shown schematically in figure 1. Assume that, at time $t$, the pores are all initially elliptical in shape, with possibly different areas and aspect ratios, and with centroids at the points

$$
\left\{z_{j}(t)=x_{j}(t)+\mathrm{i} y_{j}(t) \mid j=1, \ldots, N\right\} .
$$

Consider the $n$th pore and introduce a parametric $\zeta$-plane. Assume that the spatially uniform pressure inside the $n$th pore is $p_{B}^{(n)}(t)$. The conformal mapping from the interior of the circle $|\zeta|=1$ to the region exterior to the $n$th pore takes the form

$$
z_{n}(\zeta, t)=z_{n}(t)+\frac{\alpha_{n}(t)}{\zeta}+\beta_{n}(t) \zeta
$$

where $\alpha_{n}(t) \in R$ while $z_{n}(t), \beta_{n}(t) \in C$. The $\alpha_{n}(t)$ can be chosen to be real without loss of generality owing to a rotational degree of freedom of the Riemann mapping theorem. In the same way, $N$ such conformal maps (2.2) are introduced - one to 
describe each of the $N$ pores. The state of the system is then fully described by the parameters

$$
\left\{z_{j}(t), \alpha_{j}(t), \beta_{j}(t) \mid j=1, \ldots, N\right\} .
$$

These parameters encode all information regarding the position of the centroid of each ellipse, its area, orientation and eccentricity.

The outer flow seen by the $n$th pore due to the presence of the other $N-1$ pores is modelled by an irrotational flow with complex potential

$$
g(z, t)=\sum_{\substack{j=1 \\ j \neq n}}^{N} \frac{m_{j}(t)}{2 \pi} \log \left(1-\frac{z}{z_{j}(t)}\right) .
$$

The source/sink strength $m_{j}(t)$ is given by the rate of change of area of the $j$ th pore yielding the following relationship between $m_{j}(t)$ and its associated conformal mapping parameters:

$$
m_{j}(t)=\frac{\mathrm{d}}{\mathrm{d} t}\left(\pi\left(\alpha_{j}(t)^{2}-\left|\beta_{j}(t)\right|^{2}\right)\right)
$$

Taylor expanding the derivative of (2.4) about $z_{n}(t)$ yields

$$
g^{\prime}(z, t)=-\sum_{\substack{j=1 \\ j \neq n}}^{N} \frac{m_{j}(t)}{2 \pi\left(z_{j}(t)-z_{n}(t)\right)}-\left(\sum_{\substack{j=1 \\ j \neq n}}^{N} \frac{m_{j}(t)}{2 \pi\left(z_{j}(t)-z_{n}(t)\right)^{2}}\right)\left(z-z_{n}(t)\right)+\ldots
$$

The $n$th pore is now assumed to be evolving in a far-field flow given by the near-field approximation (2.6) truncated at the linear term as shown. Exploiting the results of Crowdy (2003a), it is known that under evolution the conformal map continues to have the form (2.2) with time-evolving parameters $z_{n}(t), \alpha_{n}(t), \beta_{n}(t)$. First, we ensure that the centroid of the $n$th pore moves with the local non-self-induced velocity so that

$$
\frac{\mathrm{d} \bar{z}_{n}}{\mathrm{~d} t}=-\sum_{\substack{j=1 \\ j \neq n}}^{N} \frac{m_{j}(t)}{2 \pi\left(z_{j}(t)-z_{n}(t)\right)} .
$$

Next, the evolution of $\alpha_{n}(t)$ and $\beta_{n}(t)$ is that relevant to a compressible elliptical pore evolving in a straining flow with strain rate $k_{n}(t)$ given by

$$
k_{n}(t)=-\sum_{\substack{j=1 \\ j \neq n}}^{N} \frac{m_{j}(t)}{2 \pi\left(z_{j}(t)-z_{n}(t)\right)^{2}} .
$$

From Crowdy (2003a), the evolution equations are then

$$
\begin{gathered}
\dot{\alpha}_{n}=-\alpha_{n} I\left(0, \alpha_{n}, \beta_{n}\right)-\left(\frac{p_{\infty}^{(n)}(t)-p_{B}^{(n)}(t)}{2}\right) \alpha_{n}, \\
\dot{\beta}_{n}=-\beta_{n} I\left(0, \alpha_{n}, \beta_{n}\right)+\left(\frac{p_{\infty}^{(n)}(t)-p_{B}^{(n)}(t)}{2}\right) \beta_{n}+2 \bar{k}_{n}(t) \alpha_{n},
\end{gathered}
$$

where

$$
I\left(0, \alpha_{n}, \beta_{n}\right)=\frac{1}{4 \pi \mathrm{i}} \oint_{\left|\zeta^{\prime}\right|=1} \frac{\mathrm{d} \zeta^{\prime}}{\zeta^{\prime}} \frac{1}{\left|z_{n \zeta}\left(\zeta^{\prime}, t\right)\right|}
$$




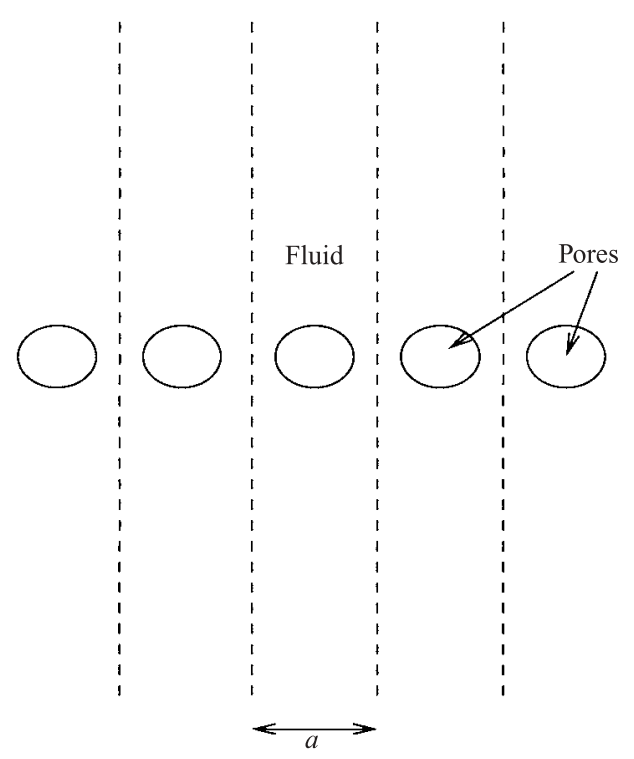

FIGURE 2. A singly periodic row of identical pores. The size of a typical period cell is $a$.

and where $p_{\infty}^{(n)}(t)$ is the 'inner limit' near $z_{n}(t)$ of the pressure field associated with the irrotational flow (2.6). However, this is zero in Stokes flow. The evolution equations for $\alpha_{n}(t)$ and $\beta_{n}(t)$ are then (2.9) and (2.10) with $p_{\infty}^{(n)}(t)=0$. Also, $p_{B}^{(n)}(t)$ will be some function of $\alpha_{n}(t)$ and $\beta_{n}(t)$ determined by the specified equation of state for the gas in the pores.

The equations above provide a closed system of coupled nonlinear ordinary differential equations for the parameters $\left\{z_{j}(t), \alpha_{j}(t), \beta_{j}(t) \mid j=1, \ldots, N\right\}$ describing the current state of the system of interacting elliptical pores.

\section{Test problem: singly periodic row of pores}

As a test problem for the model, consider a periodic array of identical pores placed along the $x$-axis. See figure 2 for a schematic. It will be assumed that the initial pores are circular. We isolate a particular period cell in the $z$-plane and refer to this as the principal period cell. This period cell is taken to be the region $0 \leqslant x \leqslant a$ where $a$ is a positive constant. Given the initial shapes and the symmetries of the configuration, it is expected that subsequent pore shapes will have reflectional symmetry with respect to horizontal and vertical axes through the centre-point $z_{c}(t)$. Given these symmetries, it is to be expected that at all times in the evolution

$$
\operatorname{Re}\left[z_{c}(t)\right]=\frac{a}{2}
$$

That is, the centre of the pore is constant in time and coincides with the centre of the principal period cell.

Let the velocity field of the planar fluid region outside the pores be $\boldsymbol{u}=(u, v)$ and let the associated fluid pressure be $p$. The flow is incompressible, so a streamfunction $\psi$ can be introduced such that

$$
\boldsymbol{u}=(u, v)=\left(\psi_{y},-\psi_{x}\right)
$$


where subscripts denote partial derivatives. The fluid vorticity $\omega$ is related to $\psi$ by $\omega=-\nabla^{2} \psi$, and $\psi$ satisfies the biharmonic equation

$$
\nabla^{4} \psi=0
$$

the general solution of which can be written in terms of the two functions $f(z, t)$ and $g(z, t)$ (Langlois 1964) as follows:

$$
\psi=\operatorname{Im}[\bar{z} f(z, t)+g(z, t)] .
$$

If there are no singularities in the flow, $f(z, t)$ and $g(z, t)$ are analytic everywhere in the flow region.

In terms of the Goursat functions, the physical field variables can be written as follows (Langlois 1964):

$$
\begin{gathered}
\frac{p}{\mu}-\mathrm{i} \omega=4 f^{\prime}(z, t), \\
u+\mathrm{i} v=-f(z, t)+z \bar{f}^{\prime}(\bar{z}, t)+\bar{g}^{\prime}(\bar{z}, t), \\
e_{11}+\mathrm{i} e_{12}=z \bar{f}^{\prime}(\bar{z}, t)+\bar{g}^{\prime}(\bar{z}, t)
\end{gathered}
$$

where

$$
e_{i j}=\frac{1}{2}\left(\frac{\partial u_{i}}{\partial x_{j}}+\frac{\partial u_{j}}{\partial x_{i}}\right)
$$

is the fluid rate-of-strain tensor; $\bar{f}(z, t)$ is defined as $\overline{f(\bar{z}, t)}$.

On each pore interface the tangential component of the fluid stresses must vanish while the normal component must be balanced by surface tension. This can be written as

$$
-p n_{i}+2 \mu e_{i j} n_{j}=\sigma \kappa n_{i}
$$

where $n_{i}$ denotes the $i$ th component of the normal vector to the interface, $\kappa$ is the curvature and $\sigma$ is the surface tension. It is convenient to non-dimensionalize lengths with respect to $r_{0}$ (a typical length scale associated with the pore), times with respect to $r_{0} \mu / \sigma$, velocities with respect to $\sigma / \mu$ and pressure with respect to $\sigma / r_{0}$. Having done this, (3.9) can be written in complex form using the relations (3.5)-(3.7). If $s$ denotes arclength around the pore boundary, it can be shown that the resulting equation can be integrated (with respect to the arclength $s$ ) to give

$$
f(z, t)+z \bar{f}^{\prime}(\bar{z}, t)+\bar{g}^{\prime}(\bar{z}, t)=-\mathrm{i} \frac{z_{s}}{2}+\frac{p_{B}(t)}{2} z+C(t)
$$

where $C(t)$ is some function of time arising from the spatial integration. The kinematic condition at the interface is that the normal velocity of the interface equals the normal fluid velocity. This can be written

$$
\operatorname{Im}\left[z_{t} \bar{z}_{s}\right]=\operatorname{Im}\left[(u+\mathrm{i} v) \bar{z}_{s}\right], \quad \text { on the interface. }
$$

\section{Application of the elliptical-pore model}

The general elliptical pore model in $\S 2$ was presented for a finite collection of pores; however it is easily extendible to a singly periodic array of pores. Assume all pores evolve through a sequence of ellipses. By the periodicity it is then enough to consider the pore in the principal period cell where the effect of all other pores is modelled by an infinite line of sinks, each of strength $m(t)$, equispaced along the $x$-axis. In this section, it is convenient to assume that the pore of interest is centred 
between $-a / 2<x \leqslant a / 2$. By the assumed symmetries of the flow, the centroids of the pores are expected to remain fixed in time. Correspondingly, let the sinks - each of strength $m(t)$ - be at fixed positions $\pm n a$ where $n \geqslant 1$. This is the model 'outer flow' which will provide the far-field boundary conditions to an 'inner flow' near the elliptical pore.

The irrotational outer flow model described above is given by

$$
g(z, t)=\frac{m(t)}{2 \pi} \sum_{n=1}^{\infty} \log \left(1-\frac{z^{2}}{n^{2} a^{2}}\right), \quad f(z, t)=0 .
$$

Taking a derivative and expanding this about the origin, $g^{\prime}(z, t)$ has a local expansion

$$
g^{\prime}(z, t) \sim k(t) z+\ldots
$$

where the (real) strain rate $k(t)$ is given by

$$
k(t) \equiv-\left(\frac{m(t)}{\pi a^{2}} \sum_{n=1}^{\infty} \frac{1}{n^{2}}\right) .
$$

The map $z(\zeta, t)$ from the interior of a unit $\zeta$-circle to the fluid exterior to the pore takes the form

$$
z(\zeta, t)=\frac{\alpha(t)}{\zeta}+\beta(t) \zeta
$$

The initially circular bubble of radius $a_{0}$ corresponds to the initial conditions

$$
\alpha(0)=a_{0}, \quad \beta(0)=0 .
$$

The evolution equations for $\alpha(t)$ and $\beta(t)$ for a bubble of spatially uniform pressure $p_{B}(t)$, with ambient pressure $p_{\infty}(t)$ in the presence of a pure time-dependent straining flow with strain rate $k(t)$ are given by

$$
\begin{gathered}
\dot{\alpha}=-\alpha I(0, \alpha, \beta)-\left(\frac{p_{\infty}(t)-p_{B}(t)}{2}\right) \alpha, \\
\dot{\beta}=-\beta I(0, \alpha, \beta)+\left(\frac{p_{\infty}(t)-p_{B}(t)}{2}\right) \beta+2 k(t) \alpha,
\end{gathered}
$$

where

Taking

$$
I(0, \alpha, \beta)=\frac{1}{4 \pi \mathrm{i}} \oint_{\left|\zeta^{\prime}\right|=1} \frac{\mathrm{d} \zeta^{\prime}}{\zeta^{\prime}} \frac{1}{\left|z_{\zeta}\left(\zeta^{\prime}, t\right)\right|}
$$

$$
p_{\infty}(t)=p_{B}(t)=0
$$

(for the case of pure sintering driven solely by surface tension with no pressure differential between the bubble and the surroundings) and

$$
m(t)=\frac{\mathrm{d}}{\mathrm{d} t}\left(\pi\left(\alpha^{2}-\beta^{2}\right)\right)=2 \pi(\alpha \dot{\alpha}-\beta \dot{\beta}),
$$

we obtain a closed system of coupled nonlinear ordinary differential equations for $\alpha(t)$ and $\beta(t)$ given, after some rearrangement, by

$$
\begin{gathered}
\dot{\alpha}=-\alpha I(0, \alpha, \beta), \\
\dot{\beta}=-I(0, \alpha, \beta)\left(\frac{\beta a^{2}+2 \Gamma \alpha^{3}}{a^{2}+2 \Gamma \alpha \beta}\right),
\end{gathered}
$$




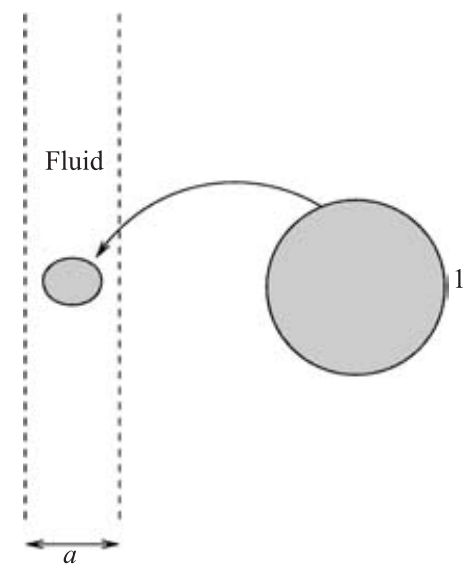

FIGURE 3. Schematic of the conformal mapping from the interior of a unit circle in a parametric $\zeta$-plane to the interior of the pore (in the principal period cell) in the physical plane.

where the constant $\Gamma$ is

$$
\Gamma=-2 \sum_{n=1}^{\infty} \frac{1}{n^{2}} .
$$

Note that the model takes into account both the surface tension effects on the pore boundary (which leads to shrinkage) as well as interaction effects with the other pores.

The simplicity of the model prompts an examination of its range of validity by comparison with numerical simulations of the full system.

\section{Boundary integral simulation}

The evolution of a singly periodic row of pores has not been treated by previous authors and a numerical method to tackle it has not been formulated in the literature. One possibility is to adapt the methods devised by Van de Vorst (1993) and Pozrikidis (2003) to study a doubly periodic array of pores. However, here we choose to present a new numerical method based on analytic functions.

\subsection{Tracking the interface: conformal mapping}

Once the instantaneous velocity field has been determined (i.e. once $f(z, t)$ and $g(z, t)$ are known), (3.11) can be used to update the pore interface position. One method is to use a distribution of Lagrangian markers on the interface; however we elect to use a spectral method based on conformal mapping.

Let $z(\zeta, t)$ be a time-dependent conformal mapping from the interior of a unit $\zeta$-circle to the interior of the pore in the principal period cell. See figure 3 for a schematic. If $z_{c}(t)$ denotes the centre-point of the principal period cell on the real axis then, using a degree of freedom of the Riemann mapping theorem, it can be assumed that

$$
z(0, t)=z_{c}(t)
$$


$z(\zeta, t)$ is also analytic everywhere in the unit $\zeta$-circle and therefore has a Taylor expansion of the form

$$
z(\zeta, t)=z_{c}(t)+\sum_{n=1}^{\infty} a_{n}(t) \zeta^{n} .
$$

The numerical method will track the evolution of the interface by computing the evolution of the time-dependent Taylor expansion coefficients in (5.2). Truncating this series at order $N$ implies that the set of coefficients to be determined is

$$
\left\{a_{n}(t) \mid n=1, \ldots, N\right\} .
$$

The reflectional symmetries of the configurations in the horizontal and vertical axes through $z_{c}$ imply that the coefficients (5.3) will be real and that $a_{n}=0$ for $n$ even.

On the pore interface, simple geometrical considerations can be used to show that

$$
z_{s}=-\frac{\mathrm{i} \zeta z_{\zeta}(\zeta, t)}{\left|z_{\zeta}(\zeta, t)\right|}
$$

Using this, and combining the stress condition (3.10) with the expression given in (3.6) for the velocity implies that (3.11) takes the form

$$
\operatorname{Re}\left[\frac{z_{t}}{\zeta z_{\zeta}}\right]=-\frac{1}{2\left|z_{\zeta}(\zeta, t)\right|}+\operatorname{Re}\left[\frac{-2 f(z)+p_{B}(t) z / 2+C(t)}{\zeta z_{\zeta}}\right] .
$$

Equation (5.5) will be used to update the coefficients in the Taylor expansion of the conformal map. Only the function $f(z, t)$ is needed for this. The calculation of $f(z, t)$ is discussed in the next two subsections.

\subsection{Properties of $f(z, t)$}

At each instant, $f(z, t)$ and $g^{\prime}(z, t)$ satisfy (3.10) which determines each function up to an additive constant. Finding these functions is identical to a problem arising in the theory of plane elasticity (cf. Mikhlin 1957; Greengard, Kropinski \& Mayo 1996). Despite the $a$-periodicity of the physical flow quantities, $f(z, t)$ and $g^{\prime}(z, t)$ are not necessarily $a$-periodic. The purpose of this subsection is to establish a decomposition of each in terms of $a$-periodic functions.

By (3.5) and the fact that both the fluid pressure $p$ and $\omega$ are $a$-periodic functions, it is deduced that

$$
f^{\prime}(z+a, t)=f^{\prime}(z, t)
$$

i.e. $f^{\prime}(z)$ is $a$-periodic. By integration with respect to $z$,

$$
f(z+a, t)=f(z, t)+\beta(t)
$$

where $\beta(t)$ is some (complex) function of time. Suppose that the pressure at infinity tends to some function of time $p_{\infty}(t)$ while the vorticity decays to zero. From (3.5),

$$
f^{\prime}(z, t) \sim \frac{p_{\infty}(t)}{4} \quad \text { as } \quad z \rightarrow \infty .
$$

Equivalently,

$$
f(z, t) \sim \frac{p_{\infty}(t)}{4} z+O(1) \quad \text { as } \quad z \rightarrow \infty .
$$

Now define a new function $f_{0}(z, t)$ via

$$
f(z, t) \equiv \frac{p_{\infty}(t)}{4} z+f_{0}(z, t) .
$$


Then, $f(z, t)$ satisfies the necessary condition (5.7) provided we choose

$$
\beta(t)=\frac{p_{\infty}(t) a}{4},
$$

and provided $f_{0}(z, t)$ satisfies

$$
f_{0}(z+a, t)=f_{0}(z, t)
$$

that is, provided $f_{0}(z, t)$ is an $a$-periodic function (that is bounded at infinity).

Next, (3.6) and the fact that the velocities are $a$-periodic means that

$$
-f(z+a, t)+(z+a) \bar{f}^{\prime}(\bar{z}+a, t)+\bar{g}^{\prime}(\bar{z}+a, t)=-f(z, t)+z \bar{f}^{\prime}(\bar{z}, t)+\bar{g}^{\prime}(\bar{z}, t) .
$$

This implies that $g^{\prime}(z, t)$ must satisfy

$$
g^{\prime}(z+a, t)=g^{\prime}(z, t)+\beta(t)-a f^{\prime}(z, t) .
$$

Now define $g_{0}^{\prime}(z, t)$ via

$$
g^{\prime}(z, t)=g_{0}^{\prime}(z, t)-z f^{\prime}(z, t)+\frac{p_{\infty}(t)}{4} z .
$$

Using (5.11), then the necessary condition (5.14) is satisfied provided

$$
g_{0}^{\prime}(z+a, t)=g_{0}^{\prime}(z, t),
$$

i.e. provided that $g_{0}^{\prime}(z, t)$ is also $a$-periodic. Finally, using conditions (5.7), (5.12) and (5.14) in (3.7) it then follows that the fluid stresses $e_{11}+\mathrm{i} e_{12}$ are also $a$-periodic.

Substituting the expressions (5.10) and (5.15) into (3.10) we obtain a boundary value problem for the two $a$-periodic functions $f_{0}(z, t)$ and $g_{0}^{\prime}(z, t)$. This takes the form

$$
f_{0}(z, t)+(z-\bar{z}) \bar{f}_{0}^{\prime}(\bar{z}, t)+\bar{g}_{0}^{\prime}(\bar{z}, t)=-\frac{\mathrm{i}}{2} z_{s}+\left(\frac{p_{B}(t)-p_{\infty}(t)}{2}\right) z+C(t) .
$$

Equation (5.17) is a boundary value problem to be solved for the two $a$-periodic functions $f_{0}(z, t)$ and $g_{0}^{\prime}(z, t)$. Once $f_{0}(z, t)$ is known, $f(z, t)$ follows from $(5.10)$.

\subsection{Integral equation for $f_{0}(z, t)$}

It is convenient to introduce a conformal mapping to facilitate the computation of $f_{0}(z, t)$. This second conformal mapping is not related to that already introduced in $\S 3.1$ to track the interface. To solve $(5.17)$ for $f_{0}(z, t)$, we first map the domain in the $z$-plane to a $\tau$-plane via a conformal mapping

$$
\tau=\exp \left(\frac{2 \pi \mathrm{i} z}{a}\right)
$$

which maps the strip $0 \leqslant x \leqslant a$ to the $\tau$-plane cut along the positive real $\tau$-axis. Figure 4 shows a schematic. This maps the pore boundary to some closed contour $L$ in the $\tau$-plane. Under evolution, this contour evolves in time; however, for brevity, we suppress the explicit time-dependence of this contour. Note also that any two points (shown as $A$ and $B$ in figure 4 ) that are at equal $y$-stations map to the same point on the real $\tau$-axis.

Defining $\Phi(\tau, t)$ and $\Psi(\tau, t)$ as

$$
\Phi(\tau, t) \equiv f_{0}(z(\tau, t), t), \quad \Psi(\tau, t) \equiv g_{0}^{\prime}(z(\tau, t), t),
$$




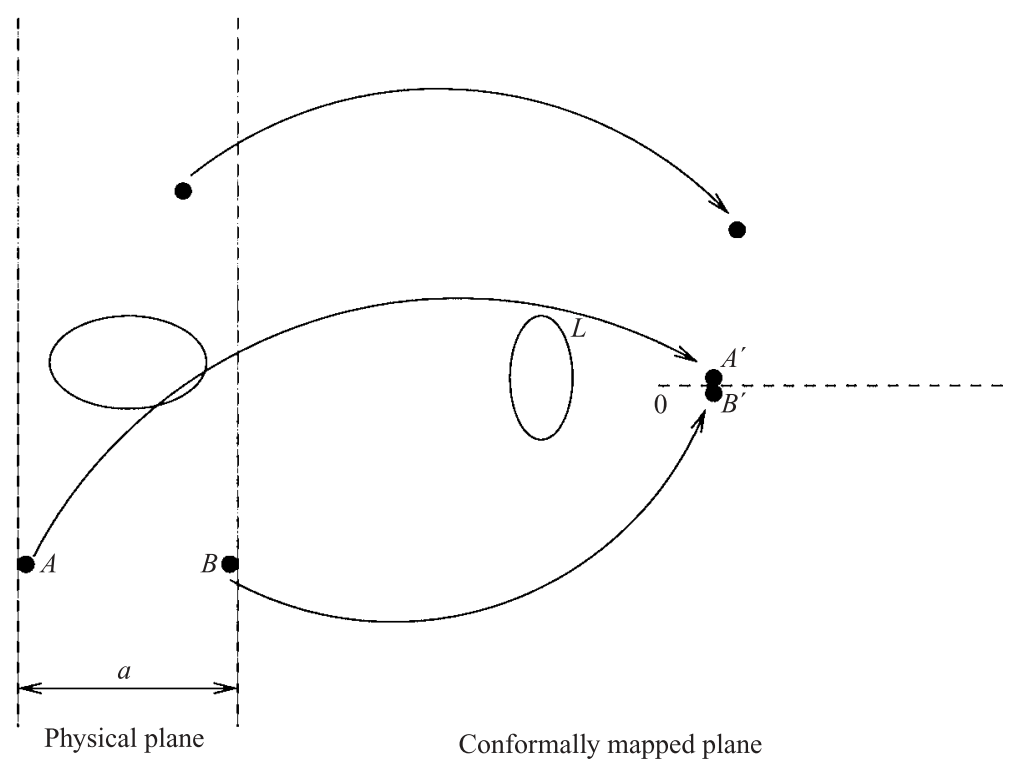

FIGURE 4. Schematic illustrating conformal mapping from the principal period cell of the physical $z$-plane to the $\tau$-plane. The pore boundary maps to a contour $L$. The two sides of the period cell both map to the positive real $\tau$-axis.

the boundary condition (5.17) assumes the form

$$
\Phi(\tau, t)-2 \bar{\tau} \log |\tau| \overline{\Phi_{\tau}(\tau, t)}+\overline{\Psi(\tau, t)}=R(\tau, t)+\left(\frac{p_{B}(t)-p_{\infty}(t)}{2}\right) z+C(t)
$$

where $R(\tau, t)$ denotes the quantity $-\frac{1}{2} \mathrm{i} z_{s}$ written as a function of $\tau$. It is satisfying that the inhomogeneous forcing term on the right-hand side of (5.20) depends only on the difference $p_{B}(t)-p_{\infty}(t)$ because, physically, we expect the evolution to depend only on the difference between the bubble pressure and the ambient pressure at infinity.

Spencer \& Meiron (1994), who were interested in calculating steady stress-induced deformations of a two-dimensional elastic interface, derived the following non-singular integral equation for $\Phi\left(\tau_{0}, t\right)$ :

$$
\begin{array}{r}
\overline{\Phi\left(\tau_{0}, t\right)}+\frac{1}{2 \pi \mathrm{i}} \oint_{L} \overline{\Phi(\tau, t)} \mathrm{d}\left[\log \frac{\tau-\tau_{0}}{\bar{\tau}-\bar{\tau}_{0}}\right]+\frac{1}{\pi \mathrm{i}} \oint_{L} \Phi(\tau, t) \mathrm{d}\left[\frac{\tau \log |\tau|-\tau_{0} \log \left|\tau_{0}\right|}{\tau-\tau_{0}}\right] \\
+\frac{1}{2 \pi \mathrm{i}} \oint_{L} \frac{\overline{\Phi(\tau, t)}}{\bar{\tau}-\bar{c}} \mathrm{~d} \bar{\tau}-\frac{1}{2 \pi \mathrm{i}} \bar{\tau}_{0} \operatorname{Re}\left[\oint_{L} \frac{\Phi(\tau, t)}{(\tau-c)^{2}} \mathrm{~d} \tau\right]=A\left(\tau_{0}, t\right)
\end{array}
$$

where

$$
A\left(w_{0}, t\right)=\frac{1}{2 \pi \mathrm{i}} \oint_{L}\left(\overline{R(\tau, t)}+\bar{C}(t)+\frac{p_{B}(t)-p_{\infty}(t)}{2} \bar{z}\right) \frac{\mathrm{d} \tau}{\tau-w_{0}}
$$

\subsection{Numerical method}

The main steps of the method are as follows. The integral equation (5.21) is solved for $\Phi(\tau, t)$. This implies that $f_{0}(z, t)$ is known on the interface. From $(5.10), f(z, t)$ is then known on the interface. This is then used in (5.5) to time-advance the conformal mapping coefficients $\left\{a_{n}(t)\right\}$. 
To solve (5.21) for $\Phi(\tau, t)$, we pick $\mathscr{N}$ points on the unit $\zeta$-circle with arguments equally spaced between 0 and $2 \pi$, convert all integrals to integrals with respect to $\theta$ and discretize them using the trapezoidal rule. The solution is found by direct inversion. The principal part integrals involved in evaluating $A\left(\tau_{0}, t\right)$ are computed using the alternate-point trapezoidal rule. The point $c$ is picked, arbitrarily, to be

$$
c(t)=\frac{1}{2}[z(\mathrm{i}, t)-z(-\mathrm{i}, t)] .
$$

Equation (5.21) is first solved by setting $C(t)=0$. For pore shapes with the reflectional symmetries described above, we expect the corresponding solution for $f(z, t)$ to be symmetric with respect to the $z$-origin so that, having computed a solution $\Phi(\tau, t)$ with $C=0$, we then let

$$
\tilde{\Phi}(\tau, t)=\Phi(\tau, t)-\frac{1}{\mathscr{N}} \sum_{j=1}^{\mathscr{N}} \Phi\left(\tau_{j}, t\right),
$$

which is equivalent to taking

$$
C(t)=\frac{1}{\mathscr{N}} \sum_{j=1}^{\mathscr{N}} \Phi\left(\tau_{j}, t\right)
$$

in (5.20). Under this transformation, the solution (5.24) is found to have the required symmetry properties with respect to the $z$-origin.

Once the solution for $f_{0}(z, t)$ satisfying all the required symmetry properties is determined, the right-hand side of (5.5) can be evaluated on the unit circle; (5.5) takes the form

$$
\operatorname{Re}\left[\frac{z_{t}-\left[p_{B}(t)-p_{\infty}(t)\right] z / 2-C(t)}{\zeta z_{\zeta}}\right]=-\frac{1}{2\left|z_{\zeta}(\zeta, t)\right|}-\operatorname{Re}\left[\frac{2 f_{0}(z)}{\zeta z_{\zeta}}\right],
$$

where we have used (5.10). The function in the square brackets on the left-hand side of $(5.26)$ is analytic everywhere in the unit $\zeta$-circle provided

$$
\dot{z}_{c}(t)-\left[p_{B}(t)-p_{\infty}(t)\right] \frac{z_{c}}{2}-C(t)=0 .
$$

The Poisson integral formula can then be used to deduce that, for $|\zeta|<1$,

$$
z_{t}(\zeta, t)=\zeta z_{\zeta}(\zeta, t) I(\zeta, t)+\left[p_{B}(t)-p_{\infty}(t)\right] \frac{z}{2}+C(t)
$$

where

$$
I(\zeta, t)=\frac{1}{2 \pi \mathrm{i}} \oint_{\left|\zeta^{\prime}\right|=1} \frac{\mathrm{d} \zeta^{\prime}}{\zeta^{\prime}}\left(\frac{\zeta^{\prime}+\zeta}{\zeta^{\prime}-\zeta}\right)\left[-\frac{1}{2\left|z_{\zeta}\left(\zeta^{\prime}, t\right)\right|}-\operatorname{Re}\left[\frac{2 f_{0}\left(z\left(\zeta^{\prime}, t\right), t\right)}{\zeta^{\prime} z_{\zeta}\left(\zeta^{\prime}, t\right)}\right]\right]
$$

is an analytic function in $|\zeta|<1$. Note that, once $C(t)$ is known, (5.27) provides an evolution equation for $z_{c}(t)$. Any imaginary part of $C(t)$ is discarded; it generates an inconsequential (time-dependent) translation of the pore in the $y$-direction. Such translations do not affect the shape evolution. It is found that with the real part of $C(t)$ obtained from (5.25) then (5.27) implies $\dot{z}_{c}=0$. This is as expected; $z_{c}(t)=\frac{1}{2} a$ under evolution, as anticipated in (3.1).

To compute $I(\zeta, t)$ on the unit circle, the Plemelj formula is used to write $I(\zeta, t)$ as a half-residue contribution plus a principal part integral which is computed numerically using the alternate-point trapezoidal rule. Having evaluated the right-hand side of 
(5.28) at $\mathscr{N}$ points (equally spaced in arclength) on the unit $\zeta$-circle, a discrete Fourier transform is used to determine its Taylor series coefficients. In order to avoid unacceptable aliasing errors, $\mathscr{N}$ was taken to be at least $8 N$. Comparison of coefficients in (5.28), yields the time derivatives of the expansion coefficients of $z(\zeta, t)$. These expansion coefficients are updated using a simple forward Euler scheme.

\subsection{Checks on the numerical procedure}

Several checks on the numerical procedure are possible. First it is checked that, for sufficiently large $N$ and $\mathscr{N}$, the solution obtained does not depend on the choice of $c(t)$ (cf. (5.23)). It is also checked that the solution does not change significantly as $N$ and $\mathscr{N}$ are independently increased. To provide a non-trivial check on the numerical solution for $\Phi(\tau, t)$, an exact solution of Chiu \& Gao (1993) to a problem in plane elasticity is employed. This exact solution is also used by Spencer \& Meiron (1994). The solution of Chiu \& Gao (1993) involves a periodic cycloidal interface. However, such a geometry involves a very simple adaptation of the numerical method described here (see Spencer \& Meiron 1994 for a discussion). Making these simple adaptations to the above code permits comparison of the numerical solution with the exact solution. For a cycloidal surface described parametrically by

$$
z=x+\mathrm{i} y=\theta+\mathrm{i} \alpha \mathrm{e}^{-\mathrm{i} \theta}
$$

for any fixed $\alpha \leqslant 1$, the elastic strain energy is known to be

$$
S(\theta)=\left(\frac{1-\alpha^{2}}{1+2 \alpha \cos \theta+\alpha^{2}}\right)^{2} .
$$

Moreover, in terms of the solution $\Phi$, the value of $S(\theta)$ at any point $\theta=\theta_{j}$, i.e. $S_{j}=S\left(\theta_{j}\right)$, is given as

$$
S_{j}=\left[1+4 \operatorname{Re}\left(\left.\mathrm{i} \tau_{j} \frac{\mathrm{d} \Phi}{\mathrm{d} \theta}\right|_{j}\left(\left.\frac{\mathrm{d} \tau}{\mathrm{d} \theta}\right|_{j}\right)^{-1}\right)\right]^{2} .
$$

Comparison of (5.32) - computed numerically - with the analytical formula (5.31) provides a check on the numerical solution.

Another check on the code is afforded by the known rate of shrinkage of a single isolated bubble, which should be retrieved in the limit that the bubble is small compared to the width of the period cell. Hopper (1990) showed that the radius of an isolated circular bubble whose internal pressure is equal to the ambient pressure at infinity shrinks at a constant rate. With the present non-dimensionalization, this rate of decrease is 0.5 .

The contour $L$ in the $\tau$-plane is typically found to be a cardioid-like shape centred on the negative $\tau$-axis. Initial pores with larger radius are expected to have a greater interaction with their nearest neighbours. This is manifested in the $\tau$-plane by the near-cuspidal region of the cardioid-like shape developing a larger curvature for initial pores with larger radius. A convenient feature of the conformal mapping approach is that it is found to naturally crowd points at the high-curvature regions of the cardioid-like $L$ where they are needed most.

It is worth mentioning that the numerical method proposed here to study the singly periodic array of pores could be combined with the fast multipole methods of Greengard et al. (1996) which also use a decomposition of the streamfunction (in twodimensional Stokes flow) in terms of two Goursat functions. At present, however, the theory of Greengard et al. (1996) is restricted to domains of finite connectivity and 

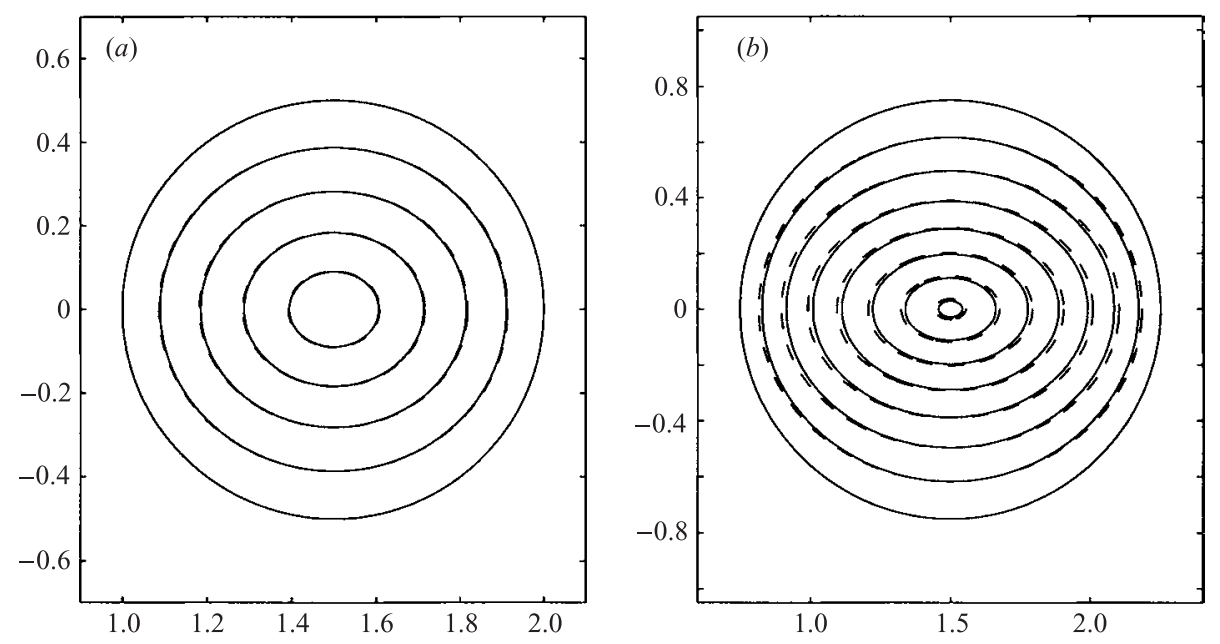

FiguRE 5. Superposition of the results of the full numerical simulation (solid lines) and the model approximation (dashed lines) for initial pores of radius $(a) r_{0}=0.5$ and $(b) r_{0}=0.75$. The time interval between successive plots is 0.2 .

generalization to the domains with infinite connectivity considered here has yet to be made. Furthermore, minor modification of the numerical method presented here can be used to compute the periodic Jeong-Moffatt flow considered by Pozrikidis (1997) using different methods. The modification required is similar to that considered in Spencer \& Meiron (1994) who compute the periodic stress distribution in a semiinfinite elastic solid.

\section{Calculations}

\subsection{Pure sintering}

Mackenzie \& Shuttleworth (1952) discuss the influence on sintering of the enclosed gas in the pores. They consider two cases in detail: the case where the pressure inside the pores is constant and the case where there is a constant mass of (ideal) gas inside each pore. Both will now be considered here.

In this section we set $p_{B}(t)=p_{\infty}(t)$ and, without loss of generality, we take both to be zero. One physical situation to which this corresponds is 'open-pores' sintering where the motion is driven purely by surface tension and not by any differentials in pressure between the bubble and its surroundings. The pores can be thought of as being open to the atmosphere. Another physical situation in which it is reasonable to assume that the pressure inside the pores is constant is when the gas in the pores can dissolve in the compact in such a way that equilibrium is maintained between the gas in the pores and that in the fluid.

In all calculations which follow, we take $a=3$. The initial radius of the circular pores is denoted $r_{0}$. To compare with the model, figure 5 features a superposition of the successive pore shapes, at time intervals of 0.2 , for pores of initial radii $r_{0}=0.5$ and 0.75 . These calculations are performed with $N=32$ and $\mathscr{N}=16 N$. The aspect ratio of the pore is found to decrease from unity under evolution so that the pore becomes more ellipse-like in shape. This is due to interaction effects with neighbouring pores since an isolated pore would simply shrink via a sequence of circles. In the case $r_{0}=0.5$, the shapes given by the full numerical simulation and by the elliptical pore 

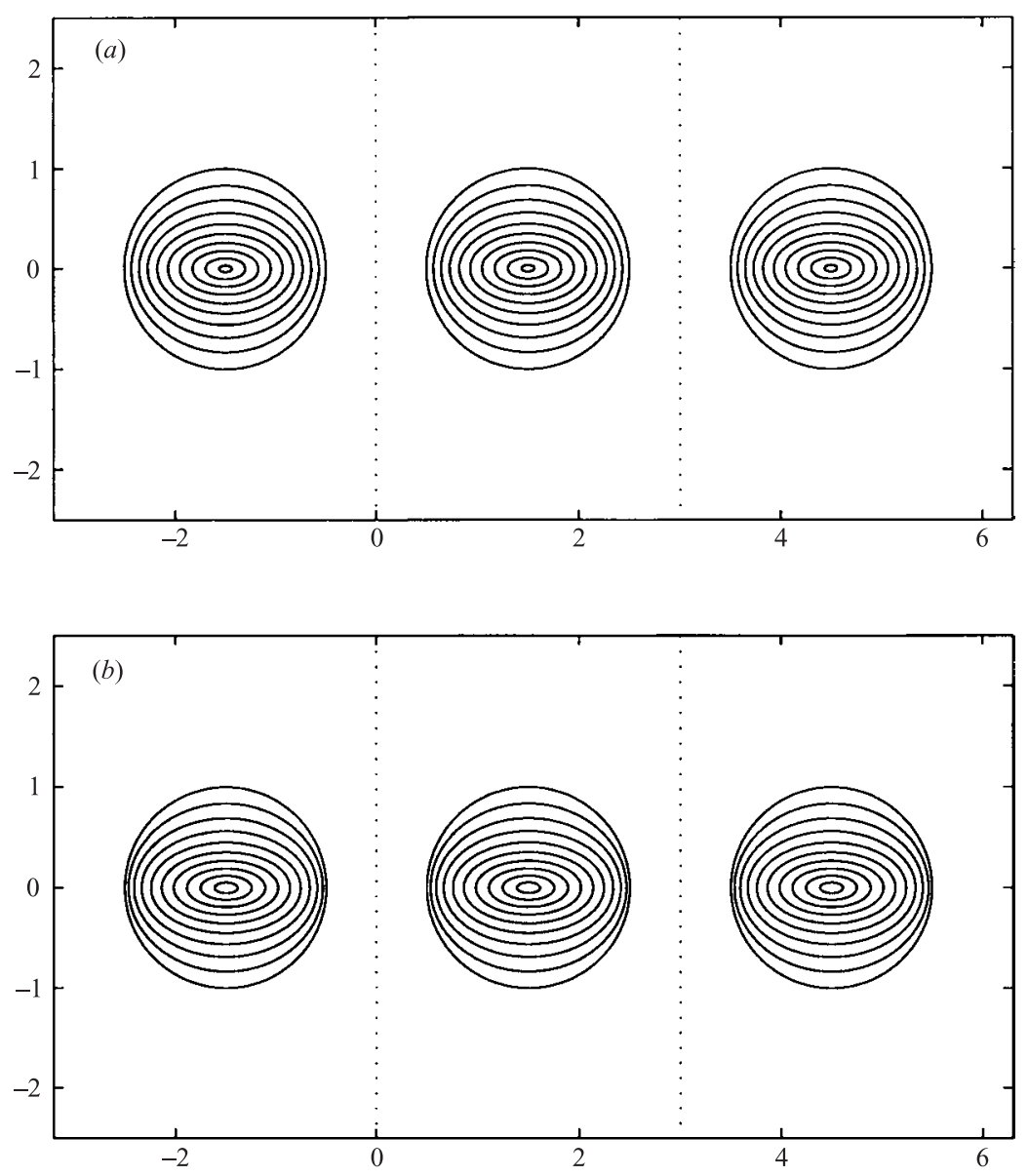

FIGURE 6. Evolution of initially circular pores with radius $=1:(a)$ the evolution as given by the full numerical simulation, $(b)$ that given by the model. Times shown are $t=0,(0.2), 1.8$.

model are almost indistinguishable. The case $r_{0}=0.75$ is critical in the sense that the pore diameter exactly equals the minimum distance between neighbouring pores and it is here that we expect the accuracy of the model to begin to break down. While the disparity between the model and the simulation indeed starts to become apparent, the model continues to provide a convincing approximation to the full shape evolution.

To further examine the validity of the model, a juxtaposition of the full numerical simulation and the model prediction is shown in figure 6 in the case where $r_{0}=1$. In this case, it should not be expected that the model will give particularly good agreement with the full simulation because the pore separation in this case is half the (initial) pore diameter. Nevertheless, at least to the eye, the respective evolutions in figure 6 are in good agreement.

As a more quantitative comparison, we follow Van de Vorst (1993) in defining the aspect ratio to be $x_{2} / x_{1}$ where $x_{1}$ and $x_{2}$ are the points at which the pore intersects the positive $x$ - and $y$-axes respectively. Figure 7 shows the evolution of this aspect ratio for pores of initial radii $r_{0}=0.5,0.75$ and 1 . The graph shows the results as computed both by the full numerical simulation and the elliptical pore model. For $r_{0}=0.5$, the model gives excellent agreement with the full simulation. This is to be 


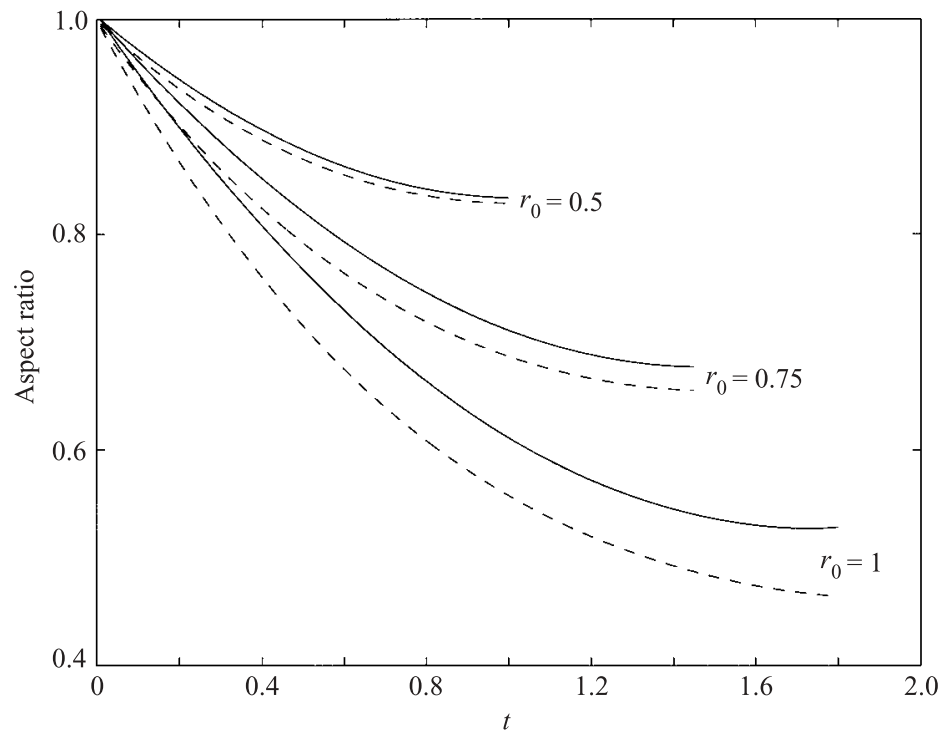

FIGURE 7. Evolution of aspect ratio $x_{2} / x_{1}$ for infinite row of circular pores with initial radii $r_{0}=0.5,0.75$ and 1 and with period $a=3$. The solid lines are the results given by the numerical simulation, the dashed lines are the results given by the elliptical pore model. As expected, the accuracy of the model deteriorates as $r_{0}$, and hence the ratio of pore diameter to minimum distance between pores, increases.

expected because, in this case, the ratio of pore diameter to the minimum distance between pores is $1 / 2$, leading to a good separation of 'inner' and 'outer' length scales the primary assumption underlying the model. In the 'critical' case $r_{0}=0.75$ the model again provides a convincing approximation to the evolution of the aspect ratio of the pores. The disparity between the graphs becomes more apparent for $r_{0}=1$, as expected since there is now no well-defined separation of inner and outer length scales. The model overestimates the rate of decrease of aspect ratio but nevertheless gives the correct qualitative trend. The pore with $r_{0}=0.5$ has little interaction with its nearest neighbours and displays an approximately linearly decreasing aspect ratio. This is in agreement with the calculations of Van de Vorst (1993) who found that an initial ellipse placed well inside a finite circular blob of fluid has little interaction with the outer boundary and its aspect ratio decreases in a near-linear fashion. Initially larger pores interact more strongly with their neighbours and have a more rapidly decreasing aspect ratio that evolves in a much less linear fashion. This is also consistent with the results of Van de Vorst (1993); indeed figure 7 should be compared to figures 6 and 8 of Van de Vorst (1993). At large times, the pores are well-separated and have shapes that are close to ellipses. Since an isolated ellipse is known to shrink with constant aspect ratio (Hopper 1990) it is not surprising that the graphs of aspect ratio against time tend to level off at large times.

While we do not expect the model to be accurate for $r_{0}$ much greater than 0.75 , it is of interest to use the boundary integral formulation to investigate the shrinkage of much larger pores. Figure 8 shows numerically computed time sequences for $r_{0}=1.4$. Larger pores are seen to interact strongly with their nearest neighbours thereby exhibiting more dramatic departures from their initial circular shapes, and even from ellipse-like shapes. The calculation in figure 8 was performed at an increased resolution with $N=64$ and $\mathscr{N}=16 N$. This is because the cardioid-type figure in the $\tau$-plane 


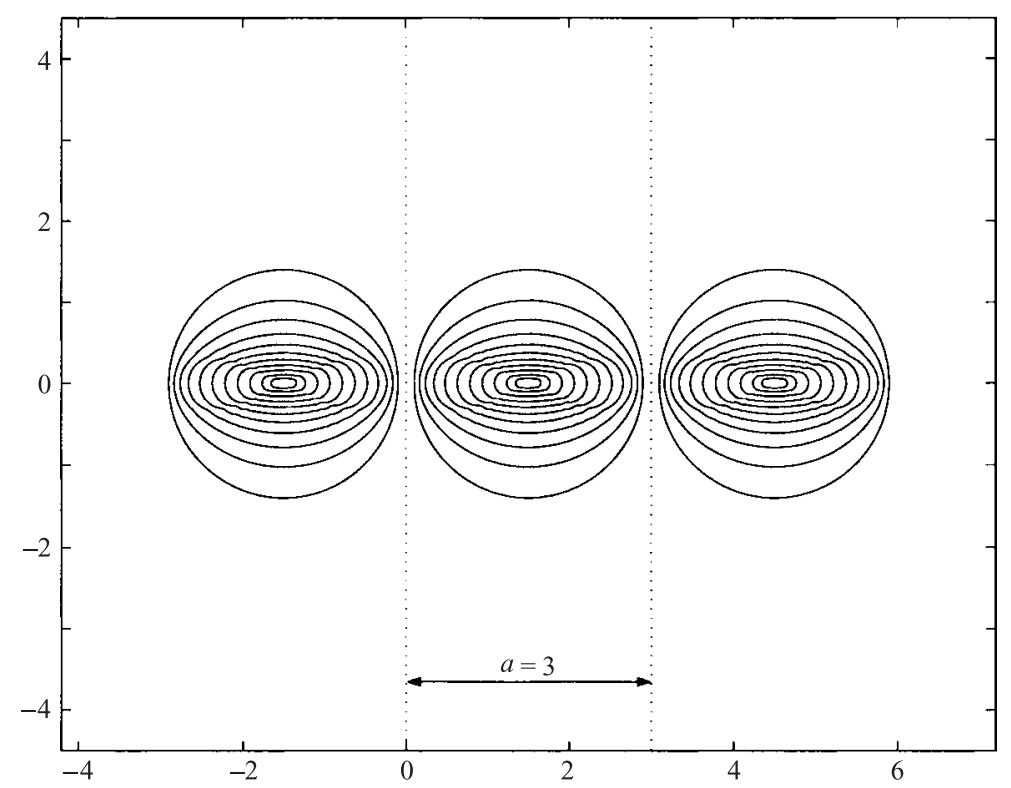

FIGURE 8 . Evolution of initially circular pores with radius $=1.4$. Times shown are $t=0,(0.2), 1.8,1.95$.

corresponding to initial radius equal to 1.4 (so that the minimum distance between pores is just 0.2 ) displayed a region of very high curvature and, for initially circular pores of even larger radius, the number of grid points used in the calculation would have to be large in order to cope with the high degree of nonlinearity associated with pore interactions.

In industrial applications, it is important to predict the time to densification, or the total pore shrinkage times. The shrinkage time for a number of initially circular pores of differing initial radius is computed: figure 9 shows the shrinkage time $t^{*}$ against initial radius. The values of $t^{*}$ reported in this graph are the times taken for the typical length scale of the pore to shrink to the order of $10^{-3}$ where the accuracy of the numerical method is found to deteriorate and the calculations are terminated. Also shown in figure 9 is the (theoretically predicted) time to full densification of the same pores under the assumption that they are isolated. This linear graph follows from the fact that the radius of an isolated circular bubble decreases at a constant rate equal to 0.5 (cf. Hopper 1991). For small initial radii, the differences in the time to densification of the periodic case from the isolated case are negligible as might be expected if interaction effects between nearest neighbours are small. However, for larger initial radii, the interaction effects with neighbouring pores are found to decrease the time to full densification. Figure 9 shows that departures from the isolated-pore shrinkage times begin to occur for $r_{0}$-values above approximately 0.75 , i.e. when the pore diameter is of the same order as the pore separation, as might be expected. Figure 9 also shows that the elliptical pore model tends to overestimate the pore shrinkage times as the initial pores draw closer together. Nevertheless, it gives the correct qualitative behaviour that shrinkage times are reduced relative to the isolated case. Indeed, it provides a more accurate model than a more simplistic one based on assuming that pore shrinkage rates in a multi-pore system are the same as those for pores in isolation. 


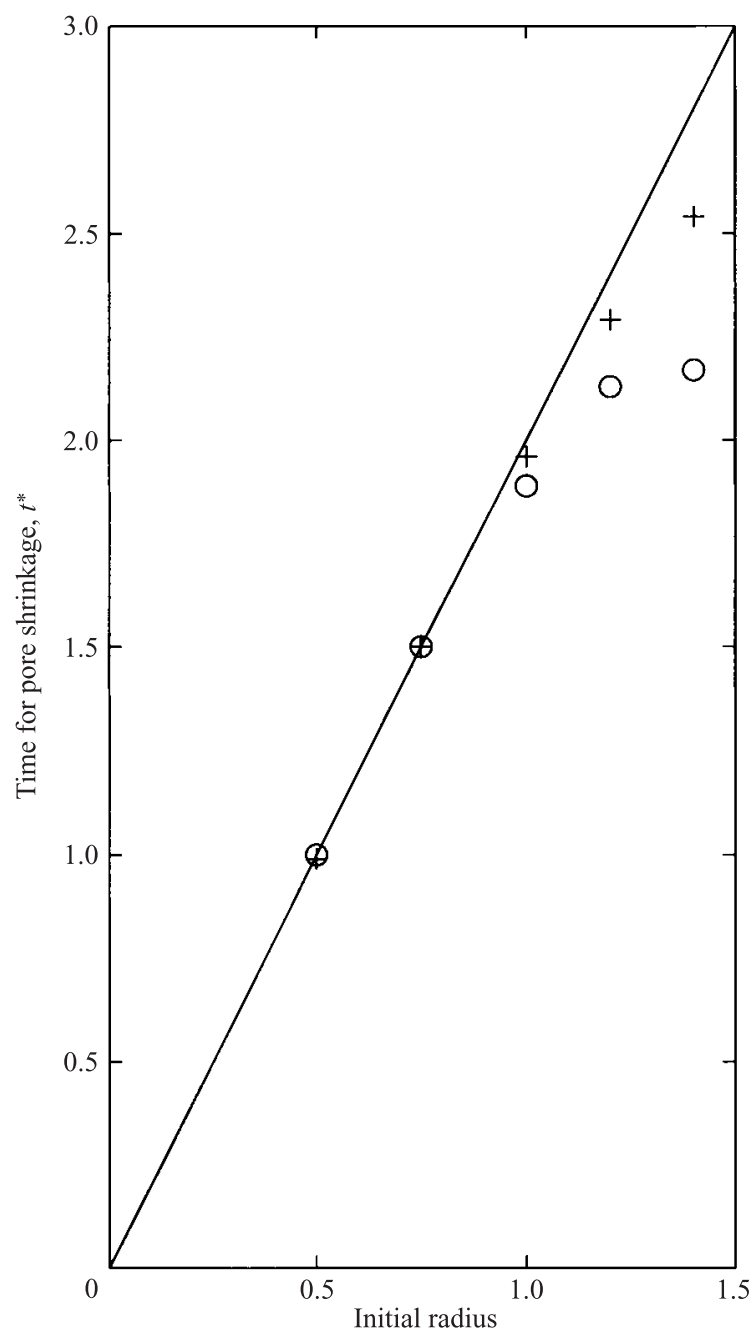

FIGURE 9. Graph of shrinkage times $t^{*}$ for an infinite row of initially circular pores with differing initial radii. The solid straight line, with slope equal to 2, gives the result for isolated pores. The circles show the results given by the full numerical simulation, the plus signs give the results predicted by the elliptical pore model.

\subsection{Sintering with compressible pores}

If an inert gas is trapped in, say, a metal compact it will not dissolve appreciably during sintering and the mass of gas inside a pore can be considered constant. Here only the hydrostatic effects of the pressure of this trapped gas will be taken into account. The pressure of the gas in the pore will obey some equation of state relating it to the pore area. Pozrikidis $(2000,2003)$ and Crowdy $(2003 b)$ have considered the problem of computing the evolution of isolated compressible bubbles in Stokes flow. These studies are now extended to the case of a singly infinite row of pores containing a fixed amount of compressible gas.

Assume that the pores contain a compressible gas obeying a pressure-area relation given by the ideal gas law. The gas is taken to be isothermal so that if $A_{B}(t)$ denotes 


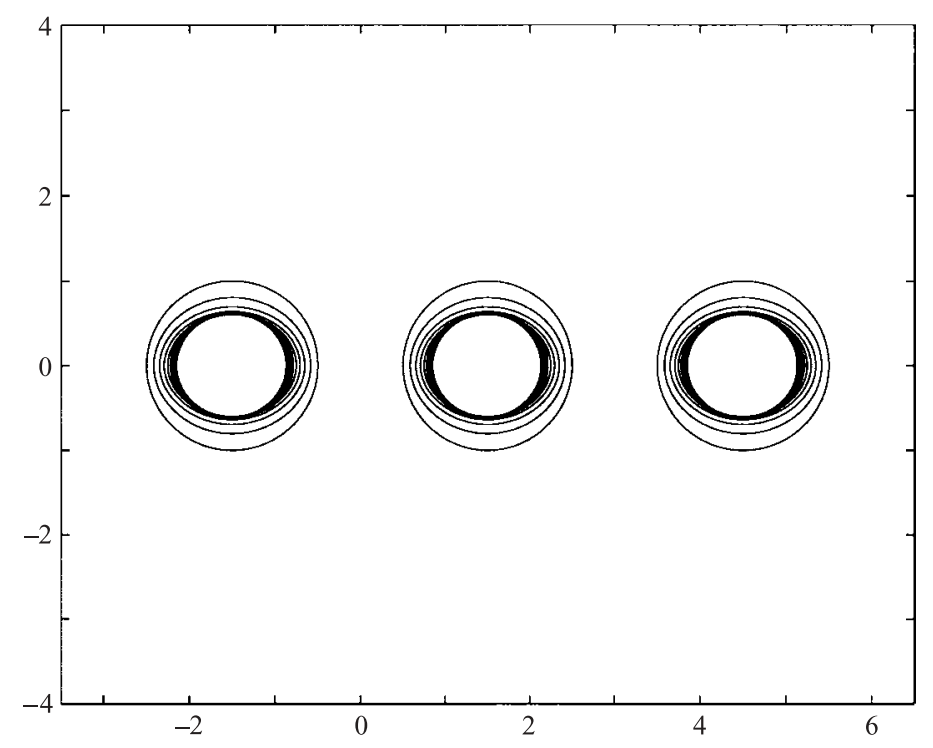

FIGURE 10. Evolution of pores containing ideal gas at constant temperature. Initial radius is 1.0. Times shown are $0,(0.3), 3.0$.

the area of one of the pores, then $p_{B}(t)$ is related to $A_{B}(t)$ via

$$
p_{B}(t) A_{B}(t)=p_{B}(0) A_{B}(0) \text {. }
$$

It is necessary to make some assumption about the ambient pressure. Motivated by the sintering application, it is assumed that $p_{\infty}(t)$ is constant in time and equal to the initial bubble pressure, i.e.

$$
p_{\infty}(t)=p_{B}(0) \text {. }
$$

Physically, this means that at the initial instant there is no pressure differential between the pore and the surroundings. The initial bubble radius is taken to be unity.

The pore area $A_{B}(t)$ is easily computed using the formula

$$
A_{B}(t)=\frac{1}{2} \operatorname{Im}\left[\oint_{|\zeta|=1} \bar{z}\left(\zeta^{-1}, t\right) z_{\zeta}(\zeta, t) \mathrm{d} \zeta\right]=\pi \sum_{j=1}^{N} j a_{j}^{2} .
$$

Figure 10 shows the evolution of three representatives of an infinite row of initially circular pores of unit radius. Again, the aspect ratio of the pores decreases initially. As time evolves, it is found that the configuration evolves to what appears to be a quasi-steady state. This occurs because the initially unbalanced capillary pressure causes the pore area to decrease. This, in turn, causes the hydrostatic pressure exerted by the gas to increase until it becomes commensurate with the capillary pressure. Figure 11 shows the evolution for a row of slightly larger circular pores of radius 1.2. A quasi-steady state is again reached, the greater interaction with neighbouring pores leading to quasi-steady pores with a more distorted shape. In the general case of compressible pores involving a fixed mass of insoluble gas, the sintering would tend asymptotically to some equilibrium density depending on the initial pore size and initial pressure difference (here assumed to be zero) between the pore and the imposed pressure at infinity. 


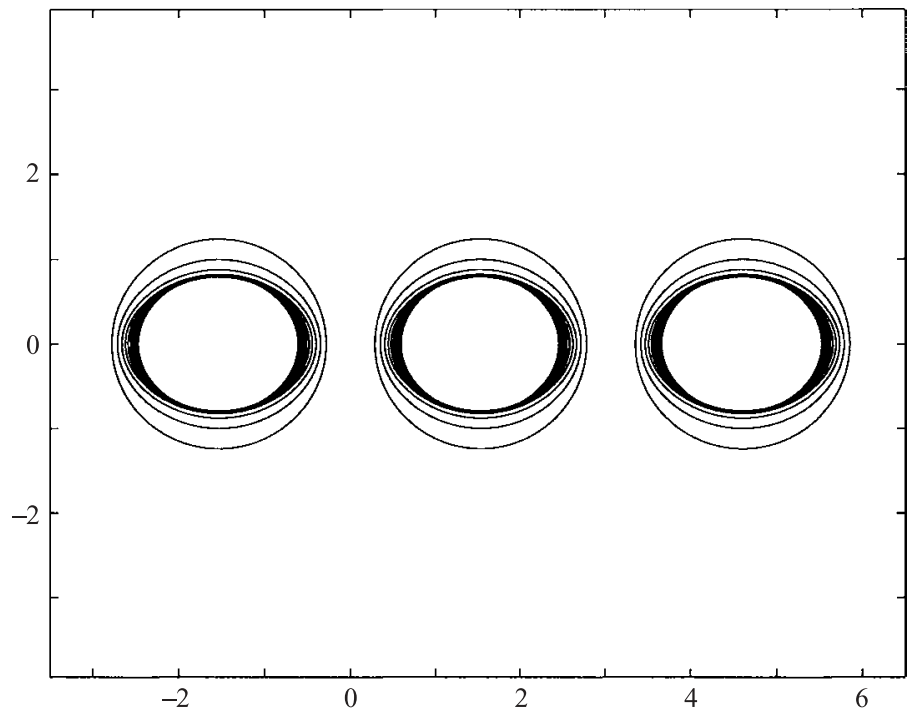

FIGURE 11. Evolution of pores containing ideal gas at constant temperature. Initial radius is 1.2 . Times shown are $0,(0.3), 3.0$.

\section{Higher-order models}

The basic idea underlying the elliptical pore model is rather general and can be extended to higher-order models. Consider now the scenario of a doubly periodic array of pores. Such a configuration is a natural model of an array of gaseous pores in a sintering medium. Pozrikidis (2003) has recently considered precisely such a problem involving doubly periodic arrays of gaseous inclusions in both square and hexagonal packings. This conveniently affords us the opportunity to pose our higher-order model and immediately test its effectiveness against the recent numerical boundary integral calculations of Pozrikidis (2003).

A schematic of the configuration in the case of a square packing is shown in figure 12. The size of each square unit cell is time-dependent, with the length of each side given by $a(t)$. A new feature of this situation is that the evolution of $a(t)$ must now also be determined as part of the solution.

A schematic of the generalized model for the square packing is shown in figure 13 . Any given pore is modelled, in an inner asymptotic sense, as a single isolated pore in a polynomially singular (i.e. fourth-order) irrotational straining flow due to the outer flow assumed to be generated by a doubly periodic array of equal point sinks of strength $m(t)$. It is easy to deduce that the irrotational outer flow is given by

$$
u-\mathrm{i} v=g^{\prime}(z, t) \sim k_{N}(t) z^{N-1} \text { as } \quad z \rightarrow \infty
$$

where $k_{N}(t)$ is the appropriate strain rate and $N=4$ (for the square packing) or $N=6$ (for the hexagonal packing). A formula for $k_{N}(t)$ is given below.

The inner problem can be solved exactly by generalizing the exact solutions of Antanovskii (1994) to the case of an arbitrarily compressible bubble. This can be done in the same way that Crowdy (2003b) generalized the exact solutions of Tanveer \& Vasconcelos (1995) to the case of compressible bubbles. We omit the details and simply state the final governing equations. It can be shown that, with far-field conditions given by (7.1) and with an initially circular pore, the conformal 


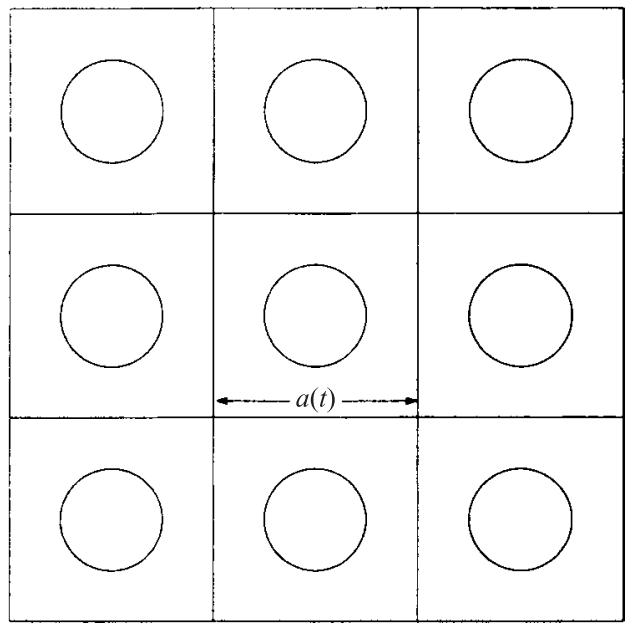

FIGURE 12. Schematic of a doubly periodic square array of pores. The length of each side of the unit cell is $a(t)$.

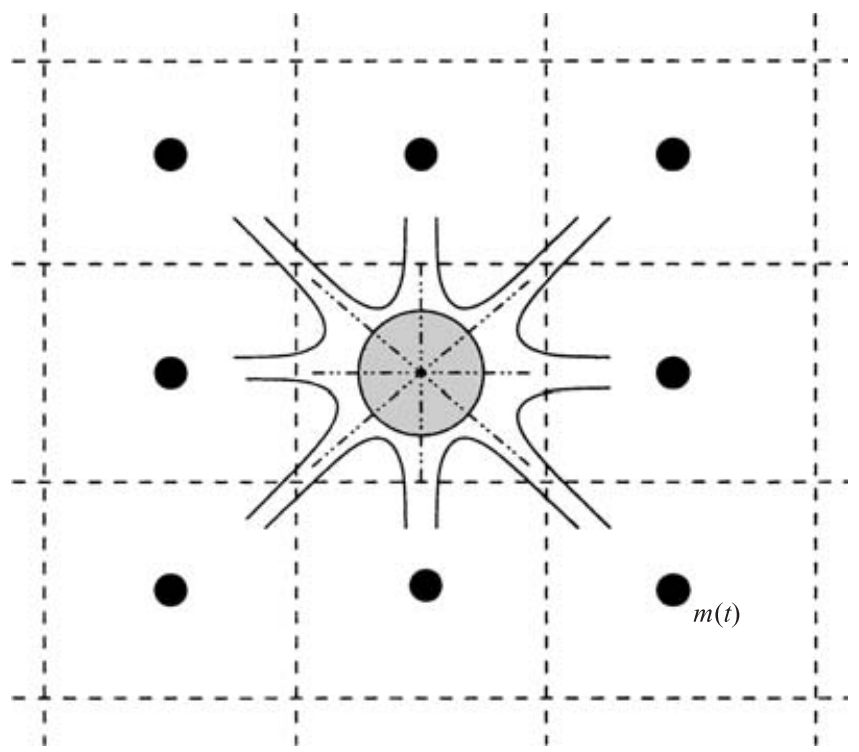

FIGURE 13. Schematic of a 'higher-order' model of a doubly periodic square array of pores. The bold dots indicate point sinks. Any given pore evolves as if placed in a fourth-order polynomial straining flow.

map from the interior of the unit circle to the exterior of the pore is of the following generalized form under evolution:

$$
z(\zeta, t)=\frac{\alpha(t)}{\zeta}+\beta(t) \zeta^{N-1}
$$

where $N=4$ (square) or $N=6$ (hexagonal); $\alpha(t)$ and $\beta(t)$ are real parameters. The evolution equations following from the generalized model are as follows. The sink 
strength $m(t)$ is equal to the rate of change of bubble area so that

$$
m(t)=\frac{\mathrm{d}}{\mathrm{d} t}\left(\pi\left(\alpha^{2}-(N-1) \beta^{2}\right)\right) .
$$

By conservation of mass, the rate of change of area of each unit cell must equal $m(t)$ so that

$$
\dot{a}=\frac{m}{2 a(t)} \quad \text { if } N=4, \quad \dot{a}=\frac{m}{\sqrt{3} a(t)} \quad \text { if } N=6 .
$$

Equations (7.4) give evolution equations for $a(t)$. The strain rate $k_{N}(t)$ is given by

$$
k_{N}(t)=-\frac{m(t) N}{2 \pi a(t)^{N}} \sum_{n=1}^{\infty} \sum_{l=0}^{\infty} \frac{1}{\left(n+l \mathrm{e}^{2 \pi \mathrm{i} / N}\right)^{N}} .
$$

The evolution equations for $\alpha(t)$ and $\beta(t)$ are then

$$
\begin{gathered}
\dot{\alpha}=-\alpha I(0, \alpha, \beta)-\left(\frac{p_{\infty}(t)-p_{B}(t)}{2}\right) \alpha \\
\dot{\beta}=-(N-1) \beta I(0, \alpha, \beta)+\left(\frac{p_{\infty}(t)-p_{B}(t)}{2}\right) \beta+2 k_{N}(t) \alpha^{N-1} .
\end{gathered}
$$

Using (7.3) and (7.5) in (7.7), combined with (7.4), now leads to a coupled system of three ordinary differential equations for the evolution of the parameters $a(t), \alpha(t)$ and $\beta(t)$.

In the case of pure sintering, we make the choice

$$
p_{B}(t)=p_{\infty}(t)=0 \text {. }
$$

The governing ordinary differential equations are then given explicitly as

$$
\begin{gathered}
\dot{\alpha}=-\alpha I(0, \alpha, \beta), \\
\dot{\beta}=-I(0, \alpha, \beta)\left(\frac{(N-1) \beta a^{N}+N \Gamma_{N} \alpha^{N+1}}{a^{N}+N(N-1) \Gamma_{N} \alpha^{N-1} \beta}\right), \\
\dot{a}=-\frac{\delta_{N} \pi}{a} I(0, \alpha, \beta)\left[\alpha^{2}-(N-1) \beta\left(\frac{(N-1) \beta a^{N}+N \Gamma_{N} \alpha^{N+1}}{a^{N}+N(N-1) \Gamma_{N} \alpha^{N-1} \beta}\right)\right],
\end{gathered}
$$

where

$$
\Gamma_{N}=-2 \sum_{n=1}^{\infty} \sum_{l=0}^{\infty} \frac{1}{\left(n+l \mathrm{e}^{2 \pi \mathrm{i} / N}\right)^{N}},
$$

and where $\delta_{N}=1$ if $N=4$ and $\delta_{N}=2 / \sqrt{3}$ if $N=6$.

Figure 14 shows the evolution, according to this model, of initially circular pores in which the initial pore radius is 0.45 of the initial half-lattice size, $a(0) / 2$. This choice is made to facilitate comparison with the numerical simulations of Pozrikidis (2003) who considered exactly this problem using numerical boundary integral methods. Figure 14 should be compared with figure 8 of Pozrikidis (2003). The figures are in close agreement suggesting that the above ordinary differential equations provide a good model of pore shrinkage in such doubly periodic configurations. Figure 15 shows the evolution of the liquid fraction calculated according to the model. As noted by Pozrikidis (2003), there is significantly more shape distortion in the square array than in the hexagonal array - a probable result of the greater degree of spatial anisotropy in the square case. This explanation is consistent with the even greater 

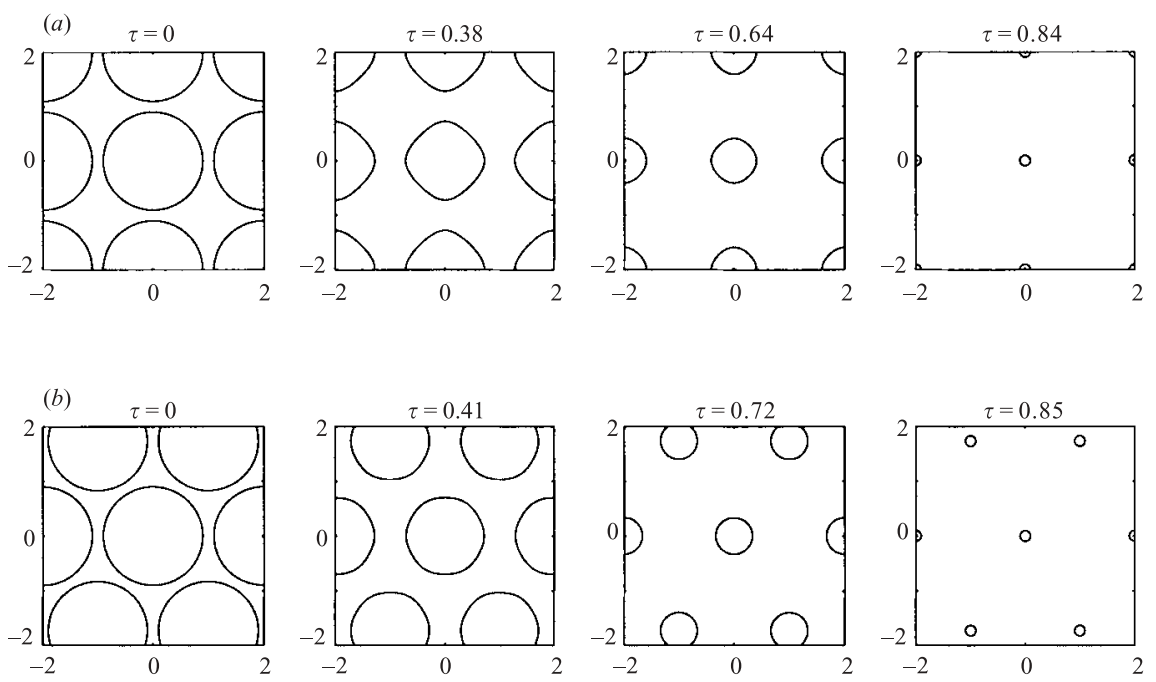

FIGURE 14. Evolution (according to the model) of shrinking constant-pressure pores on $(a)$ a square and $(b)$ hexagonal lattice. The initial pore radius is 0.45 of the initial half-lattice size $a(0) / 2$ and $\tau$ is a dimensionless time. These figures should be compared with those computed using full numerical simulations by Pozrikidis (2003, figure 8).

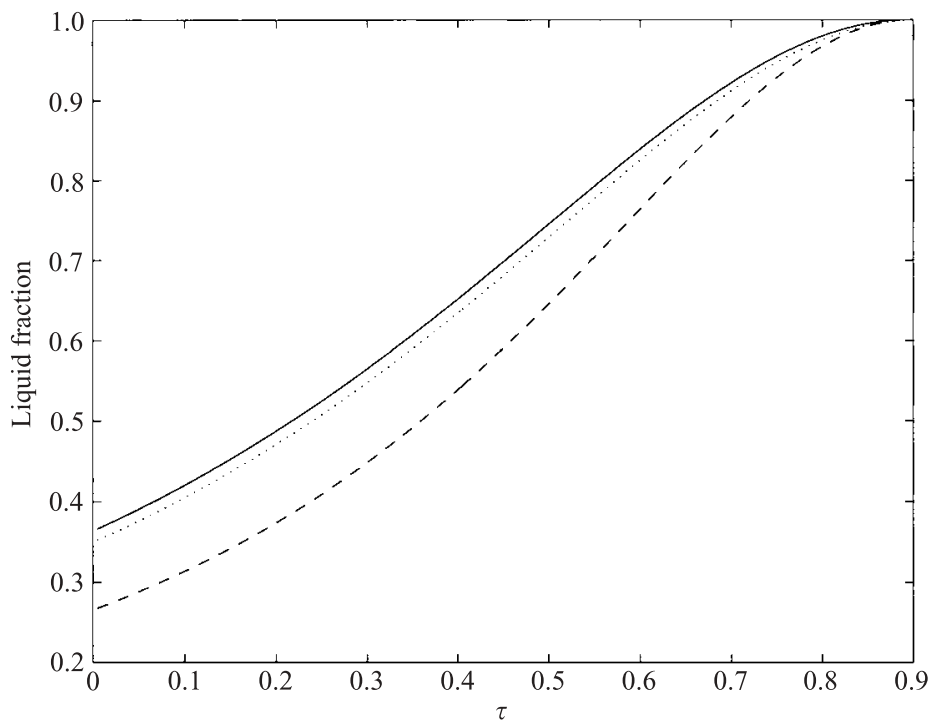

FIGURE 15. Evolution (according to the model) of the liquid fraction during densification due to shrinkage of constant-pressure pores. The solid and dashed lines correspond, respectively, to the square and hexagonal calculations shown in figure 14. The dotted line corresponds to a square lattice with initial liquid fraction equal to 0.35 . This figure should be compared with a similar graph plotted using full numerical simulations by Pozrikidis (2003, figure 9).

degree of shape distortion in the singly periodic case where there is more spatial anisotropy than in the square doubly periodic configuration. In all calculations, it is also observed that the pores become more circular as their area shrinks. Compressible pores, with non-zero $p_{B}(t)$, can be modelled in the same way. 


\section{Discussion}

A model, based on exact solutions, for the interaction of multiple pores (or bubbles) in planar Stokes flows has been presented and tested by comparison with full numerical simulations in some example cases. The basic idea is to split a multipore system into a collection of 'inner problems' in the neighbourhood of any given pore of interest, all other pores being modelled by an assembly of point sinks. Assuming the pores are not too close together, the net effect of all other pores in the 'inner' region close to any given pore is to induce an irrotational, time-dependent, straining flow which can affect both the rate of pore shrinkage and the evolution of its shape. The advantage of this lies in the fact that a wide class of exact solutions for an isolated compressible pore in a polynomially singular, time-dependent, potential straining flow are known (Crowdy 2003b; Tanveer \& Vasconcelos 1995; Antanovskii 1994) which can be used to exactly solve the 'inner problem' for the free-surface evolution of the pore. At the same time, microstructural information (such as the rate of pore area shrinkage) derived from solution of the inner problem gives the macroscopic parameters needed in the outer flow approximation.

The generic situation is the 'elliptical-pore model' which is relevant when the pore configuration is devoid of any spatial symmetries. It is also relevant in the case of a singly periodic array of pores and the validity of the model has been tested by devising a novel numerical method based on Cauchy potentials and conformal mapping. The simple model provides a good description of both the pore shrinkage time and the shape evolution provided the typical pore separation is larger than (or of the order of) the typical pore size. For pores that are too close together, the model is found to slightly over-estimate the pore shrinkage times. Full numerical simulations of more closely packed pores reveal that the pores disappear faster than predicted by the model and can shrink to near-zero area in up to $80 \%$ of the time needed if the pore were in isolation. Interaction with neighbouring pores therefore has the effect of decreasing the pore shrinkage times, these effects being more pronounced for pores that are initially closer together. Nevertheless, when the pores are close together - so that one does not expect the model to be accurate - it gives closer agreement to the actual pore shrinkage times than an alternative model that assumes the pores simply remain circular and shrink at a constant rate.

A well-known model of late-stage sintering is that of Mackenzie \& Shuttleworth (1952). They have proposed a phenomenological model in three dimensions where all pores are assumed spherical. Every pore of radius $r_{1}$ is assumed to be surrounded by a spherical shell, out to some radius $r_{2}$ of the real incompressible material making up the compact. Beyond $r_{2}$, it is assumed that the real material is replaced by an 'equivalent homogeneous medium' which models the combined effects of all other pores. Although our model applies only to the two-dimensional case, it is superior to that of Mackenzie \& Shuttleworth (1952) in two ways: first, it allows for nontrivial shape evolution while still being described by just a small set of time-evolving parameters whose evolution equations can be written down explicitly; second, it employs a more realistic model of the effects of other pores by using a distribution of point sinks. Extension of the basic model to the more realistic three-dimensional case is also under investigation and the viability of an analogous 'ellipsoidal-pore model' for late-stage viscous sintering is being examined. The present two-dimensional results suggest that such a model is worth investigating. Finally, the planar model presented herein has been motivated by the 'elliptical vortex approximation' used to model interacting vortical regions. It is interesting to note that the elliptical approximation 
for vortical interactions has recently been extended to a three-dimensional ellipsoidal model (Dritschel, Reinaud \& McKiver 2004).

The author is grateful to Professors C. Pozrikidis and C. Lawrence for insightful discussions. This work is supported by EPSRC Grant GR/R40104/01 and the Nuffield Foundation.

\section{REFERENCES}

AntanovskiI, L. K. 1994 Quasi-steady deformation of a two-dimensional bubble placed within a potential viscous flow. Eur. J. Mech. B 13, 73-92.

AntanovskiI, L. K. 1996 Formation of a pointed drop in Taylor's four-roller mill. J. Fluid Mech. 327, 325-341.

Brinker, C. J. \& Scherer, G. W. 1990 Sol-Gel Science.

Chiu, C. H. \& GaO, H. 1993 Stress singularities along a cycloid rough surface. Intl J. Solids. Struct. 30 (21), 2983-3012.

Crowdy, D. G. 2002 Exact solutions for the viscous sintering of multiply-connected fluid domains. J. Engng Maths 42, 225-242.

Crowdy, D. G. 2003a Viscous sintering of unimodal and bimodal cylindrical packings with shrinking pores. Eur. J. Appl. Maths 14, 421-445.

Crowdy, D. G. 2003b Compressible bubbles in Stokes flow. J. Fluid Mech. 476, 345-356.

Crowdy, D. G. \& TanveER, S. 1998 A theory of exact solutions for annular viscous blobs. J. Nonlin. Sci. 8, 375-400.

Crowdy, D. G. \& Tanveer, S. 2001 Erratum to "A theory of exact solutions for annular viscous blobs". J. Nonlin. Sci. 11, 237.

Dritschel, D. G. \& Legras, B. 1991 The elliptical model of two-dimensional vortex dynamics I: the basic state. Phys. Fluids 3, 845-854.

Dritschel, D. G., Reinaud, J. N. \& MCKiver, W. 2004 The quasi-geostrophic ellipsoidal vortex model, J. Fluid Mech. (in press).

Frenkel, J. 1945 Viscous flow of crystalline bodies under the action of surface tension. J. Phys. (Moscow) 9, 385-391.

Greengard, L., Kropinski, M. C. \& Mayo, A. 1996 Integral equations methods for Stokes flow and isotropic elasticity in the plane. J. Comput. Phys. 125, 403-414.

HiLl, A. I. 2001 Modelling the shrinkage kinetics during the late stages of viscous sintering. Masters thesis, San Diego State University.

Hopper, R. W. 1990 Plane Stokes flow driven by capillarity on a free surface. J. Fluid Mech. 213, 349-375.

Hopper, R. W. 1991 Plane Stokes flow driven by capillarity on a free surface. Part 2. Further developments. J. Fluid Mech. 230, 355-364.

KIDA, S. 1981 Motion of an elliptic vortex in a uniform shear flow. J. Phys. Soc. Japan 50, 3517-20.

KuIKEN, H. K. 1990 Viscous sintering: the surface-tension-driven flow of a liquid form under the influence of surface tension gradients at its surface. J. Fluid Mech. 214, 503-515.

Langlois, W. E. 1964 Slow Viscous Flow. Macmillan.

MacKenzie, J. K. \& Shuttleworth, R. 1952 A phenomenological theory of sintering. Proc. Phys. Soc. B 62, 833-852.

Melander, M. V., Zabusky, N. J. \& Styczek, A. S. 1986 A moment model for vortex interactions of the two-dimensional Euler equations. Part 1. Computational validation of a Hamiltonian elliptical representation. J. Fluid Mech. 167, 95-115.

Mikhlin, S. G. 1957 Integral Equations. Pergamon.

Moore, D. W. \& Saffman, P. G. 1971 Structure of a line vortex in an imposed strain. In Aircraft Wake Turbulence (ed. J. H. Olsen, A. Goldburg \& M. Rogers), Plenum Press, New York.

Newton, P. K. 2001 The N-vortex Problem. Springer.

PozRIKIDIS, C. 1997 Numerical studies of singularity formation at free surfaces \& fluid interfaces in two-dimensional Stokes flow. J. Fluid Mech. 331, 145-167.

PozRikidis, C. 1998 Numerical studies of cusp formation at fluid interfaces in Stokes flow. J. Fluid Mech. 357, 29-57. 
Pozrikidis, C. 2000 Expansion of a compressible bubble in Stokes flow. J. Fluid Mech. 442, 171-189.

PozRIKIDIS, C. 2003 Computation of the pressure inside bubbles and pores in Stokes flow. J. Fluid Mech. 474, 319-337.

Primo, A. R. M., Wrobel, L. C. \& Power, H. 2000 An indirect boundary-element method for slow viscous flow in a bounded region containing air bubbles. J. Engng Math. 37, 305-326.

Richardson, S. 1992 Two-dimensional slow viscous flows with time dependent free boundaries driven by surface tension. Eur. J. Appl. Maths 3, 193-207.

Richardson, S. 2000 Plane Stokes flow with time-dependent free boundaries in which the fluid occupies a doubly-connected region. Eur. J. Appl. Maths 11, 249-269.

Saffman, P. G. 1992 Vortex Dynamics. Cambridge University Press.

Spencer, B. J. \& Meiron, D. I. 1994 Nonlinear evolution of the stress-driven morphological instability in a two-dimensional semi-infinite solid. Acta Metall. Mater. 42, 3629-3641.

Tanveer, S. \& Vasconcelos, G. 1995 Time evolving bubbles in two dimensional Stokes flow. J. Fluid Mech. 301, 325-344.

VAN DE VORST, G. A. L. 1993 Integral method for a two-dimensional Stokes flow with shrinking holes applied to viscous sintering. J. Fluid Mech. 257, 667-689.

VAN DE VORST, G. A. L. 1995 Integral formulation to simulate the viscous sintering of a twodimensional lattice of periodic unit cells. J. Engng Math. 30, 97-118. 